\title{
Las democracias con libertades disminuidas en Latinoamérica en el siglo XXI y la Carta Democrática Interamericana: ¿Dos modelos de democracia en la región? $21^{\text {st }}$ Century illiberal democracies in Latin America and the Inter-American Democratic Charter: Two models of democracy in the region?
}

\section{DANIEL SORIA LUJÁN*}

Resumen: La Carta Democrática Interamericana (CDI) fue adoptada en el año 2001 por los Estados miembros de la Organización de Estados Americanos (OEA) como un instrumento renovado para la defensa de la democracia, no sólo contra el golpe de Estado tradicional, sino también para hacer frente a las graves violaciones de la responsabilidad horizontal. El segundo supuesto consideró, como precedente, la situación política en el Perú durante el gobierno de Alberto Fujimori (1995-2000), el cual fue definido por los académicos de Ciencias Políticas y Derecho Constitucional como un régimen autoritario competitivo. Sin embargo, durante la última década hasta la actualidad hemos hallado en América Latina varios países con gobiernos en donde el principio de equilibrio de poderes ha erosionado como resultado de las medidas adoptadas por sus respectivas ramas ejecutivas. Esta situación sugiere las siguientes preocupaciones: $i E l$ modelo democrático liberal de la CDI se encuentra en crisis? ¿Este modelo ha sido vencido por los gobiernos liberales lo cuales privilegian a los derechos económicos y sociales y restringen los derechos civiles y políticos? ¿O ambos modelos están condenados a coexistir en la región?

Palabras clave: Democracia en América Latina - OEA - UNASUR - autoritarismo competitivo - Carta Democrática Interamericana - pesos y contrapesos - Accountability horizontal - democracias con libertades disminuidas

Abstract: The Inter-American Democratic Charter (IDC) was adopted in 2001 by member states of the Organization of American States (OAS) as a renewed instrument for the defense of democracy, not only against traditional coup d'etat but also to face serious violations to horizontal accountability. The second assumption took into consideration, as a precedent, the political situation in Peru during Alberto Fujimori's administration (1995-2000), defined as a competitive authoritarian regime by Political Science and

* Abogado y magíster en Ciencia Política por la Pontificia Universidad Católica del Perú. Profesor de Derecho Constitucional en esta casa de estudios desde el año 2000. Miembro de la Asociación Peruana de Derecho Constitucional. Correo electrónico: daniel.soria@pucp.edu.pe. Ponencia presentada en el IX Congreso Mundial de la Asociación Internacional de Derecho Constitucional (Oslo, Noruega, 16-20 de junio de 2014) bajo el título « $21^{\text {st }}$ Century Illiberal Democracies in Latin America and the Inter-American Democratic Charter: Two Models of Democracy in the Region?». 
Constitutional Law scholars. However, during the last decade to the present we find in Latin America several countries with governments where the principle of checks and balances has been eroded as a result of measures adopted by their respective executive branch. This situation suggests the following concerns: The liberal democratic model of the IDC is in crisis? This model has been overcame by illiberal governments that privileges economic and social rights and restraints civil and political rights? Or both models are condemned to coexist in the region?

Key words: Democracy in Latin America - OAS - UNASUR - competitive authoritarianism - Inter American Democratic Charter-check and balanceshorizontal accountability - illiberal democracies

\begin{abstract}
CONTENIDO: I. INTRODUCCIÓN.- II. DEMOCRACIA CON ADJETIVOS EN LATINOAMÉRICA.- III. LA CARTA DEMOCRÁTICA INTERAMERICANA: SU DEFINICIÓN DE DEMOCRACIA Y SUS PROBLEMAS DE APLICACIÓN.IV. LA UNASUR Y LA DEFENSA DE LAS DEMOCRACIAS SUDAMERICANAS.V. CONCLUSIONES.- VI. BIBLIOGRAFÍA.
\end{abstract}

\title{
I. INTRODUCCIÓN
}

En una conferencia de prensa con medios de comunicación extranjeros el 21 de febrero de 2014, el presidente venezolano Nicolás Maduro acusó a los presidentes de Chile, Colombia y Panamá de ceder a la presión del Departamento de Estado de los Estados Unidos de América difundiendo opiniones contra el gobierno de Venezuela. Maduro manifestó a sus colegas lo siguiente: «Respeten la diversidad de América Latina a fin de mantener la unión [de los países latinoamericanos]». También afirmó que, por ejemplo, él no emitió juicios sobre la represión estatal contra estudiantes universitarios chilenos, quienes protestaban contra las matrículas educativas, a pesar de que él consideraba que la educación no debía tener costos, como en Venezuela.

Las palabras de Maduro fueron un requerimiento de respeto al principio internacional de no intervención. Pero, ifueron también una exigencia de respeto a lo que para él son diferentes modelos de democracia en Latinoamérica, especialmente el modelo que enfatiza los derechos económicos y sociales frente al modelo que privilegia los derechos civiles y políticos? ¿Estaría hablando entonces de dos modelos de democracia en la región?

El presente artículo es un esfuerzo académico por mostrar, una vez más, que América Latina necesita romper con su pasado y liberarse, de una vez por todas, de sus lazos autoritarios. Para tal propósito, la Carta Democrática Interamericana (OEA, 2001) podría ser la hoja de ruta hacia la democracia del siglo XXI. 


\section{DEMOCRACIA CON ADJETIVOS EN LATINOAMÉRICA}

De acuerdo con algunos politólogos, varios países de América Latina son democracias «con adjetivos», es decir, parafraseando a Abraham Lincoln, democracias del pueblo, pero no necesariamente por el pueblo ni para el pueblo. En efecto, el proceso democratizador de las últimas décadas del siglo XX produjo autoridades electas y amplia representación, pero sin accountability ${ }^{1}$ ni efectivo ejercicio de los derechos fundamentales.

Al respecto, Fareed Zakaria (1997) acunó la frase «illiberal democracy» («democracia con libertades disminuidas») ${ }^{2}$. El término «illiberal» está referido a derechos políticos (elecciones libres y justas), mientras que el término «democracy» hace referencia a dos aspectos: el Estado de derecho («rule of law») y la separación de poderes, y la protección de libertades fundamentales como las de expresión, reunión, religión y propiedad — derechos individuales que aseguran la autonomía personal frente a la coerción estatal, de la iglesia o de la sociedad-. Zakaria establece un espectro de democracias con libertades disminuidas, «oscilando entre modestos violadores como Argentina y cuasi-tiranías como Kazakstán y Bielorrusia, con países como Rumania y Bangladesh en el medio» (p. 23). Todos estos países tienen en común un nivel mínimo de participación popular a través de elecciones periódicas, pero difieren en el grado de violaciones al Estado de derecho, a la accountability, y a los derechos civiles y políticos.

La mencionada definición de Zakaria ha sido posteriormente criticada. Al respecto, Jørgen Møller afirma que se trata de una definición puramente electoral de democracia: «La denominación "democracia" por consiguiente denota la presencia de elecciones libres y justas, y nada más» (2008, p. 556). Asimismo, Steven Levitsky y Lucan A. Way consideran que clasificar regímenes híbridos como subtipos de democracia, como hizo Zakaria, es un error. Siguiendo a Juan Linz, creen que, en esos casos, es mejor añadir el adjetivo «autoritario» que «democrático» por lo que prefieren hablar de «regímenes autoritarios competitivos» (Levitsky \& Way, 2010, p. 15). Para los referidos autores, dichos regímenes

[...] son regímenes civiles en los que existen instituciones democráticas formales que son ampliamente reconocidas como medios primarios para la obtención del poder, pero en los cuales los abusos del poder

1 El término inglés accountability no es fácil de traducir al español. En este se entenderá por ella lo siguiente: un atributo de control que permite exigir a una persona o entidad la responsabilidad o rendición de cuentas por el ejercicio de sus funciones públicas.

2 Esta traducción personal del término expresa mejor, a nuestro entender, lo que manifiestan los autores citados. De otro lado, a efectos de guardar coherencia, entenderemos como «liberal democracy" (democracia liberal o régimen liberal) al régimen político en el que se goza plenamente de los derechos civiles y políticos; asimismo, la frase «libertades civiles» comprenderá, en algunos casos, tanto a los derechos civiles como a los políticos.

LAS DEMOCRACIAS

CON LIBERTADES

DISMINUIDAS EN

LATINOAMÉRICA

EN EL SIGLO

XXIY LA CARTA

DEMOCRÁTICA

INTERAMERICANA:

¿DOS MODELOS

DE DEMOCRACIA

EN LA REGIÓN?

$21^{\text {ST }}$ CENTURY

ILLIBERAL

DEMOCRACIES IN

LATIN AMERICA

AND THE INTER-

AMERICAN

DEMOCRATIC

CHARTER:TWO

MODELS OF

DEMOCRACY IN

THE REGION? 
estatal por parte de sus detentadores los ubican en una ventaja significativa frente a sus oponentes. Tales regímenes son competitivos en la medida que los partidos de oposición utilizan las instituciones democráticas para competir seriamente por el poder, pero no son democráticos porque el campo de juego está muy inclinado a favor de los detentadores del poder. La competencia es, por consiguiente, real pero injusta (p. 5).

De acuerdo con esa definición, los regímenes autoritarios competitivos tienen elecciones regulares y los partidos de oposición no están prohibidos de participar, pero se producen medidas arbitrarias contra ellos. Sin embargo, «tal abuso no es suficientemente severo o sistemático como para impedir a la oposición que realice una campaña nacional». En segundo lugar, en estos regímenes las libertades civiles están «nominalmente garantizadas y al menos parcialmente respetadas», aunque son vulneradas con acciones abiertas y sutiles, como acosos, detenciones y ataques violentos en el primer caso, o el uso de represión «legal» en el segundo caso - por ejemplo, mediante inspecciones tributarias selectivas y subsecuentes denuncias por la comisión de delitos tributarios, campañas de difamación, etcétera-. En tercer lugar, los regímenes autoritarios competitivos se caracterizan por un «terreno de juego desnivelado» en tres aspectos, todos a favor de los detentadores del poder: acceso a recursos públicos, a los medios de comunicación y a las instancias legales —en este caso a través de la manipulación de la judicatura o de los órganos electorales utilizando el chantaje, el soborno y/o la intimidación- (Levitsky \& Way, 2010, pp. 8-12).

Maxwell A. Cameron, en un reciente artículo, manifiesta una postura crítica de la literatura sobre autoritarismo competitivo, en el sentido de que este concepto «pone el estándar de democracia excesivamente alto. Toma a la democracia liberal como su punto de referencia valorativo» (2014). Este politólogo piensa, citando un artículo de 2012 de Yonatan L. Morse, que «Levitsky y Way emplean una definición muy estricta de democracia, donde cualquier violación de la imparcialidad de las elecciones, de las libertades civiles o del nivel del terreno de juego político convierte a un régimen en no democrático». Cameron pone el ejemplo de las antiguas políticas sobre derechos de las minorías en Canadá aplicadas a su pueblo aborigen y en los Estados Unidos hacia los afroamericanos. Al respecto, señala que «[el nivel de] protección de los derechos debe influenciar nuestra clasificación de una democracia como más o menos liberal, o de alta o baja calidad, pero no podemos excluir del grupo de democracias a cada país que viola cualquier derecho de las minorías». Por esta razón, concluye que «necesitamos ser claros respecto del punto de quiebre que utilizamos, justificarlo teóricamente y aplicarlo consistentemente». 
Guillermo O'Donnell es otro académico que puso un adjetivo a la palabra «democracia». Este politólogo señaló que la democracia «delegativa» «descansa en la premisa de que quien quiera que gane la elección a la presidencia está, de esta manera, autorizado a gobernar como él o ella lo vea conveniente, restringido solo por la cruda realidad de las relaciones de poder existentes y por el periodo de mandato limitado constitucionalmente». El punto clave de este tipo de democracia es la ausencia o tenue presencia de la accountability horizontal'. En palabras de O'Donnell, "la accountability horizontal, característica de la democracia representativa, es extremadamente débil o inexistente en las democracias delegativas» (1994, pp. 59-61).

En suma, las posiciones académicas que consideran a las democracias con adjetivos como una clasificación válida para categorizar a los regímenes latinoamericanos, utilizan un concepto de democracia relacionado básicamente a los derechos políticos. Apreciamos, entonces, la influencia de la definición de «poliarquía» del politólogo estadounidense Robert A. Dahl: «Un orden político con ciudadanía extendida, derechos políticos y derechos necesarios para participar en las elecciones, tales como la libertad de expresión o el acceso a la información» $(1989$, p. 221) 4 . De acuerdo con Dahl, «todas las instituciones de la poliarquía son necesarias para la máxima viabilidad del éxito del proceso democrático en el gobierno de un país». Sin embargo, precisa que «decir que todas las siete instituciones son necesarias no quiere decir que ellas son suficientes» (p. 222).

El sociólogo francés Alain Touraine tiene la misma opinión. Él considera que un mercado político abierto y competitivo no es plenamente identificable con la democracia, precisando que «no podemos contentarnos con una democracia de deliberación; necesitamos una democracia de liberación». En tal sentido, define al régimen democrático como «la forma de vida política que da la mayor libertad al mayor número, que protege y reconoce la mayor diversidad posible» (2006, pp. 15, 20, 23).

Luego de tomar nota de los conceptos y definiciones previamente mencionados, podríamos decir que, si tenemos elecciones libres y justas, y los derechos políticos y civiles relacionados con estas, entonces, tenemos un régimen democrático. Sin embargo, solo tendríamos los cimientos y el primer piso de nuestro edificio, por lo que necesitaríamos un segundo piso para conseguir el ejercicio pleno de todos los derechos

3 La accountability es horizontal porque este atributo de control se ejerce entre poderes públicos que no se encuentran en relaciones jerárquicas unos respecto de otros.

4 Dahl específicamente señala que la poliarquía es un orden político que se distingue por la presencia de siete instituciones: autoridades electas, elecciones libres y justas, sufragio inclusivo, derecho a competir por un cargo público, libertad de expresión, acceso a fuentes de información alternativas y autonomía asociativa.

LAS DEMOCRACIAS

CON LIBERTADES

DISMINUIDAS EN

LATINOAMÉRICA

EN EL SIGLO

XXI Y LA CARTA

DEMOCRÁTICA

INTERAMERICANA:

¿DOS MODELOS

DE DEMOCRACIA

EN LA REGIÓN?

$21^{\text {ST }}$ CENTURY

ILLIBERAL

DEMOCRACIES IN

LATIN AMERICA

AND THE INTER-

AMERICAN

DEMOCRATIC

CHARTER:TWO

MODELS OF

DEMOCRACY IN

THE REGION? 
civiles, políticos, económicos, sociales y culturales, así como para tener un gobierno que los proteja y promueva. En tal sentido, la primera mitad del segundo piso podría ser el resto de derechos civiles no incluidos en el primer piso, así como los derechos económicos, sociales y culturales y, en general, todo tipo de derechos fundamentales que podrían aparecer en el futuro en respuesta a nuevas formas de agresión a la dignidad humana.

Por su parte, la segunda mitad del segundo piso podría incorporar los mecanismos de control del ejercicio del poder político, como la accountability horizontal y social. Accountability horizontal es el término de la ciencia política para definir lo que en el derecho constitucional es el sistema de checks and balances y, de acuerdo con Guillermo O'Donnell, se divide en la accountability horizontal de balance y la accountability horizontal de encargo o mandato. La primera es una prerrogativa de los poderes Ejecutivo, Legislativo y Judicial, que estos ejercen uno contra otro para dejar sin efecto trasgresiones contrarias a derecho que afecten sus respectivas competencias. La segunda es un atributo de control de ciertas entidades públicas distintas a los clásicos poderes del Estado, muchas de ellas órganos constitucionales autónomos, como la Defensoría del Pueblo, consejos de Estado o contralorías, "que están legalmente encargadas de supervisar, prevenir, disuadir, promover una sanción o sancionar presuntos actos u omisiones contrarios al ordenamiento jurídico, realizados por otras entidades estatales, nacionales o subnacionales» (O'Donnell, 2003, pp. 44-45).

De otro lado, la accountability social es definida por Enrique Peruzzotti y Catalina Smulovitz como un mecanismo de control de las autoridades políticas, vertical, pero no electoral ${ }^{5}$, basado en un amplio espectro de acciones que adoptan elementos de la sociedad civil, así como medios de comunicación. Estos autores también señalan que la accountability social «está respaldada por las acciones de sectores organizados de la sociedad civil y de medios de comunicación, interesados en ejercer influencia en el sistema político y en las burocracias públicas», por ejemplo, monitoreando la conducta de los servidores públicos, exponiendo y denunciando actos ilegales de estos servidores, y activando el control horizontal de las entidades públicas (2002, pp. 32-33).

5 Las elecciones políticas son la expresión de la accountability vertical. 
De esta manera, nuestro «edificio» podría verse como esta figura:

\begin{tabular}{|c|c|c|}
\hline $\begin{array}{l}\text { democracia } \\
\text { (suficiente) }\end{array}$ & $\begin{array}{l}\text { - el resto de libertades civiles no } \\
\text { incluidas en el primer piso } \\
\text { - derechos económicos, sociales } \\
\text { y culturales } \\
\text { - derechos fundamentales del } \\
\text { futuro }\end{array}$ & $\begin{array}{l}\text { - accountability horizontal } \\
\text { - accountability social }\end{array}$ \\
\hline $\begin{array}{l}\text { democracia } \\
\text { (necesaria) }\end{array}$ & $\begin{array}{l}\text { - elecciones libres y justas, de- } \\
\text { rechos políticos (accountability } \\
\text { vertical) } \\
\text { - libertades civiles relacionadas } \\
\text { con las elecciones y los dere- } \\
\text { chos políticos en general }\end{array}$ & \\
\hline
\end{tabular}

En la vida real no podemos erigir el segundo piso antes que los cimientos y el primer piso de un edificio. A nuestro entender, este hecho físico podría ser una metáfora de la construcción de la democracia. Al respecto, algunos gobiernos latinoamericanos dan mayor importancia a la inversión en educación, salud o alimentación, pero violan derechos civiles y políticos —especialmente los de sus opositores políticos-, tales como la libertad personal, de expresión, de reunión, e incluso la integridad personal o la vida en algunos casos; asimismo, organizan elecciones parcializadas y erosionan tanto la accountability horizontal como la social. Estos países se definen a sí mismos como democracias, pero no existe deliberación o crítica sobre las políticas públicas relacionadas con el adecuado ejercicio de los derechos económicos y sociales, y tampoco se desarrollan mecanismos de control de los recursos públicos, por lo que no puede saberse si hay corrupción en las entidades públicas. Si la deliberación y el control existen, son débiles o están neutralizados por sutiles acciones gubernamentales - por ejemplo, por medio de procedimientos legales de fiscalización por funcionarios de la administración tributaria o el acoso a los opositores políticosConsecuentemente, en estos regímenes la democracia puede concebirse como aquella casa construida sobre la arena, descrita por Jesucristo, que se vino abajo luego de que descendiera la lluvia, vinieran las inundaciones y soplara el viento (San Mateo, 7, 26-27). Tarde o temprano, la falta de un pleno ejercicio de los derechos fundamentales y de la accountability demolerá esa democracia, más parecida, entonces, a un castillo de naipes.

Asimismo, una democracia con un primer piso como el referido en los párrafos precedentes, donde no hay planes para construir los pisos superiores, es una democracia incompleta, con espacio insuficiente para
13

LAS DEMOCRACIAS

CON LIBERTADES

DISMINUIDAS EN

LATINOAMÉRICA

EN EL SIGLO

XXI Y LA CARTA

DEMOCRÁTICA

INTERAMERICANA:

¿DOS MODELOS

DE DEMOCRACIA

EN LA REGIÓN?

$21^{\text {ST }}$ CENTURY

ILLIBERAL

DEMOCRACIES IN

LATIN AMERICA

ANDTHE INTER-

AMERICAN

DEMOCRATIC

CHARTER:TWO

MODELS OF

DEMOCRACY IN

THE REGION? 
incluir todas las necesidades y aspiraciones ciudadanas. Este espacio es muy importante en Latinoamérica porque aunque esta región no es la más pobre del mundo, sí es la más desigual. Así, para lograr el efectivo ejercicio de los derechos económicos y sociales, no es necesario sacrificar los derechos civiles y políticos. Libertad e igualdad no son términos contradictorios o incompatibles.

A pesar de lo anteriormente señalado, varios regímenes democráticos latinoamericanos se podrían agrupar de acuerdo con una suerte de división propia de la guerra fría, en materia de ejercicio de los derechos humanos. Sobre el particular, como recuerda Nico Schrijver, cuando la Declaración Universal de Derechos Humanos fue adoptada en 1948, «la intención original fue establecer una declaración de derechos en la forma de una trinidad: una declaración, una convención y un órgano de monitoreo. Sin embargo, poco después de la adopción de la Declaración Universal, la guerra fría empezó a intensificarse». Como consecuencia de ello, debido a la división entre el Este y el Oeste, se decidió establecer no uno, sino dos tratados para implementar la Declaración Universal (2011, pp. 257-258). De esta manera, en 1966 se crearon el Pacto Internacional de Derechos Civiles y Políticos y el Pacto Internacional de Derechos Económicos, Sociales y Culturales.

¿Qué tipo de democracia define la Carta Democrática Interamericana (OEA, 2001)? iEste documento mantiene la división de los instrumentos de derechos humanos de las Naciones Unidas? ¿Solo está relacionada con la democracia liberal?

\section{LA CARTA DEMOCRÁTICA INTERAMERICANA: SU DEFINICIÓN DE DEMOCRACIA Y SUS PROBLEMAS DE APLICACIÓN}

La Carta Democrática Interamericana (CDI) fue adoptada en Lima por la Asamblea General de la Organización de los Estados Americanos (OEA) el 11 de setiembre de 2001. Fue iniciativa del Gobierno de Transición del Perú, instalado después del escape del presidente Alberto Fujimori al Japón en noviembre de 2000. Dicho escape se dio como consecuencia de la difusión de un video que mostraba a Vladimiro Montesinos — asesor presidencial y líder, al igual que Fujimori, de una organización criminal dentro del gobierno- cometiendo un soborno a través del pago de US\$15000 en efectivo a un congresista electo de la oposición a fin de que se incorporara al grupo político fujimorista.

Para el Gobierno de Transición, esta iniciativa tenía dos objetivos fundamentales: reintegrar al Perú a la comunidad interamericana y proponer un medio efectivo que permita la acción colectiva de los países de la región en la difusa área que se ubica entre el clásico 
golpe de Estado y las medidas antidemocráticas de gobiernos legítimos (Cooper, 2004, p. 97). Es importante especificar que la CDI es un instrumento internacional que tiene una débil fuerza vinculante (soft law), principalmente porque no cuenta con cláusulas especiales relativas a su cumplimiento efectivo. El artículo 1 de la CDI señala que «los pueblos de América tienen derecho a la democracia y sus gobiernos la obligación de promoverla y defenderla». Si revisamos otros artículos de este instrumento internacional, podemos encontrar elementos de la democracia «de primer piso»:

- La CDI precisa cuáles son los elementos de la democracia representativa, los cuales incluyen, por ejemplo, el respeto a los derechos humanos y las libertades fundamentales; el acceso al poder y su ejercicio con sujeción al Estado de derecho; la celebración de elecciones periódicas, libres, justas y basadas en el sufragio universal y el secreto como expresión de la soberanía del pueblo; el régimen plural de partidos y organizaciones políticas, y la separación e independencia de los poderes públicos (artículo 3).

- Enfatiza que la libertad de expresión y de prensa son componentes fundamentales del ejercicio de la democracia (artículo 4).

- Señala que el fortalecimiento de los partidos y de otras organizaciones políticas es prioritario para la democracia (artículo 5).

- Puntualiza la importancia de la participación ciudadana como condición necesaria para el pleno y efectivo ejercicio de la democracia (artículo 6).

Asimismo, encontramos en la CDI elementos de la democracia de la primera mitad de nuestro segundo piso:

- Refiere que la democracia es esencial para el desarrollo social, político y económico de los pueblos de las Américas (artículo 1).

- Precisa que el respeto por los derechos sociales es un componente fundamental del ejercicio de la democracia (artículo 4).

- Resalta que la democracia es indispensable para el ejercicio efectivo de las libertades fundamentales y los derechos humanos, en su carácter universal, indivisible e interdependiente, consagrados en las respectivas constituciones de los Estados y en los instrumentos interamericanos e internacionales de derechos humanos (artículo 7).

- Declara que los Estados miembros reafirman su intención de fortalecer el sistema interamericano de protección de los derechos

LAS DEMOCRACIAS

CON LIBERTADES

DISMINUIDAS EN

LATINOAMÉRICA

EN EL SIGLO

XXI Y LA CARTA

DEMOCRÁTICA

INTERAMERICANA:

¿DOS MODELOS

DE DEMOCRACIA

EN LA REGIÓN?

$21^{\text {ST }}$ CENTURY

ILLIBERAL

DEMOCRACIES IN

LATIN AMERICA

AND THE INTER-

AMERICAN

DEMOCRATIC

CHARTER:TWO

MODELS OF

DEMOCRACY IN

THE REGION? 
humanos para la consolidación de la democracia en el hemisferio (artículo 8).

- Señala que la eliminación de toda forma de discriminación —especialmente la discriminación de género, étnica y racial—y de las diversas formas de intolerancia, así como la promoción y protección de los derechos humanos de los pueblos indígenas y los migrantes, y el respeto a la diversidad étnica, cultural y religiosa en las Américas contribuyen al fortalecimiento de la democracia y la participación ciudadana (artículo 9).

- Menciona que la promoción y el fortalecimiento de la democracia requieren el ejercicio pleno y eficaz de los derechos de los trabajadores y la aplicación de normas laborales básicas (artículo $10)$.

- Considera que la promoción y observancia de los derechos económicos, sociales y culturales son consustanciales al desarrollo integral, al crecimiento económico con equidad y a la consolidación de la democracia en los Estados del hemisferio (artículo 13).

Finalmente, encontramos también elementos de la democracia de la segunda mitad de nuestro segundo piso:

- Reconoce como elementos esenciales de la democracia representativa la separación e independencia de los poderes públicos (artículo 3).

- Establece que la transparencia de las actividades gubernamentales, la probidad y la responsabilidad de los gobiernos en la gestión pública son componentes fundamentales del ejercicio de la democracia (artículo 4).

Como podemos apreciar, la CDI considera a la democracia no solo como un régimen liberal que pone el acento en el disfrute de los derechos políticos (accountability vertical) y algunos derechos civiles como la libertad de reunión o de expresión. También incluye el ejercicio de los derechos económicos, sociales y culturales, así como la necesidad de accountability horizontal y social. Es decir, utilizando la metáfora bíblica anteriormente señalada, la CDI promueve la construcción de un sólido edificio multinivel edificado sobre roca, que no puede derribarse como consecuencia de la lluvia, las inundaciones y el viento (San Mateo, 7, 24-25). Ninguna división de la guerra fría se percibe en la CDI, tal como en los pactos de derechos humanos de las Naciones Unidas.

La CDI también establece procedimientos para el fortalecimiento y preservación de las instituciones democráticas. Al respecto, dos tipos de acciones pueden ser adoptadas por la OEA en el marco de este 
instrumento internacional: preventivas y correctivas. Las acciones preventivas están reguladas en los artículos 17 y 18, con relación a las siguientes situaciones:

- cuando el gobierno de un Estado Miembro considere que está en riesgo su proceso político institucional democrático o su legítimo ejercicio del poder (artículo 17);

- cuando en un Estado miembro se produzcan situaciones que pudieran afectar el desarrollo del proceso político institucional democrático o el legítimo ejercicio del poder (artículo 18).

En el primer caso, el gobierno del Estado miembro involucrado en la situación podrá recurrir al secretario general o al Consejo Permanente a fin de solicitar asistencia para el fortalecimiento y preservación de la institucionalidad democrática. En el segundo caso, el secretario general o el Consejo Permanente podrán, con el consentimiento previo del gobierno afectado, disponer visitas y otras gestiones con la finalidad de hacer un análisis de la situación. Posteriormente, el secretario general elevará un informe al Consejo Permanente y este realizará una apreciación colectiva de la situación y, en caso necesario, podrá adoptar decisiones dirigidas a la preservación de la institucionalidad democrática y su fortalecimiento. Es importante notar que la primera acción preventiva es motivada por la voluntad del gobierno y la segunda es una iniciativa propia de la OEA, pero, en ambos casos, es indispensable el consentimiento del gobierno.

El artículo 17 fue invocado, por primera vez, en el año 2009 por el presidente de Honduras, Manuel Zelaya (Legler, 2011, p. 42), debido a la oposición del Congreso, la Corte Suprema y su propio partido político a la propuesta de convocar a un referéndum para la instalación de una asamblea constituyente que cambiaría la Constitución para permitir la relección presidencial. El enviado de la OEA públicamente apoyó la iniciativa presidencial y aquellos que se opusieron al referéndum interpretaron la presencia de la OEA como un acto de legitimación de la propuesta de Zelaya. Días después, un golpe de Estado destituyó al presidente a través de la fuerza militar (Perina, 2012, p. 81).

Por otra parte, el artículo 18 también fue invocado aparentemente por gobiernos, a pesar de que esta cláusula literalmente permite a la OEA tomar iniciativa con el fin de realizar acciones preventivas. Respecto de esta práctica, Rubén M. Perina señala que «bajo esta disposición, a pedido de los gobiernos de Nicaragua (2005), Ecuador (2005 y 2010), Bolivia (2008), Guatemala (2009), Honduras (2009) y Haití (2010-2011), el Consejo Permanente y el secretario general actuaron diligente y efectivamente, aprobando las correspondientes resoluciones y enviando misiones políticas que exitosamente evitaron una crisis

LAS DEMOCRACIAS CON LIBERTADES DISMINUIDAS EN LATINOAMÉRICA EN EL SIGLO XXIY LA CARTA DEMOCRÁTICA INTERAMERICANA: ¿DOS MODELOS DE DEMOCRACIA EN LA REGIÓN?

$21^{\text {ST }}$ CENTURY

ILLIBERAL

DEMOCRACIES IN

LATIN AMERICA AND THE INTER-

AMERICAN

DEMOCRATIC

CHARTER:TWO

MODELS OF

DEMOCRACY IN

THE REGION? 
política de ruptura del orden democrático» $(2012$, p. 80). Cabe precisar que el autor señala que estas acciones de la OEA evitaron golpes de Estado, por lo que no necesariamente fueron acciones para prevenir la progresiva erosión de las instituciones democráticas.

Con relación a las acciones correctivas, el artículo 19 fue establecido para enfrentar dos situaciones:

- la ruptura del orden democrático en un Estado miembro;

- la alteración del orden constitucional que afecte gravemente el orden democrático en un Estado miembro.

La primera situación es el clásico golpe de Estado. En este caso, el artículo 21 señala que la OEA deberá impulsar gestiones diplomáticas. Si estas fracasan, la Asamblea General podrá suspender al Estado miembro en el ejercicio de su derecho de participación en la OEA, con el voto afirmativo de dos tercios de los Estados miembros. Este procedimiento fue aplicado, por primera vez, en el golpe de Estado perpetrado en Venezuela en abril de 2002 por un grupo de militares y civiles contra el presidente Hugo Chávez. Sin embargo, al final no hubo necesidad de aplicar el artículo 21 de la CDI porque Chávez fue reinstalado en el cargo algunos días después. El referido procedimiento fue aplicado, por segunda vez, siete años después (2009) como consecuencia del golpe contra Manuel Zelaya, presidente de Honduras. En este caso, la Asamblea General de la OEA suspendió a Honduras del ejercicio de sus derechos como Estado miembro. No obstante, Zelaya nunca regresó a su cargo, se llevaron a cabo nuevas elecciones, fue elegido Porfirio Lobo como nuevo presidente y, finalmente, la suspensión fue levantada.

La segunda situación es, hoy en día, más frecuente que los golpes de Estado en muchos países de América Latina. Al respecto, icómo podríamos definir la palabra «alteración»? De acuerdo con Perina, algunas alteraciones son fáciles de detectar — por ejemplo, elecciones manipuladas o actuaciones contrarias al derecho del Congreso, el Poder Judicial o de los militares contra el gobierno legítimo-, pero otras no. Este autor refiere que

[...] una «alteración» más controvertida es el proceso de incremento de la autocracia y del monopolio del poder del Poder Ejecutivo, el cual lentamente socava el proceso democrático, todo en el nombre de la democracia participativa, el socialismo o el anti-imperialismo, y utilizando medios democráticos como las elecciones o los referéndums. Este proceso de alteración por erosión comúnmente vacía de contenido y suprime instituciones democráticas esenciales, valores y prácticas tales como los pesos y contrapesos, límites al poder, respeto a la oposición política, el Estado de derecho y las libertades fundamentales, entre otras. Más específicamente, podría involucrar la remoción de jueces que 
no están políticamente alineados con el gobierno, la desobediencia de fallos judiciales o de legislación emitida por un Congreso controlado por la oposición, ignorar o manipular a los otros poderes del Estado, cerrar $\mathrm{o}$ atacar a la prensa independiente, o perseguir oponentes políticos, tal como ocurre hoy, de diversas maneras, en Bolivia, Ecuador, Nicaragua y Venezuela. La naturaleza de este fenómeno político, sin embargo, no es fácil de aceptar o condenar, un hecho que dificulta una defensa colectiva de la democracia $(2012$, p. 80$)$.

El artículo 20 de la CDI establece que, si esta situación ocurre, cualquier Estado miembro o el secretario general podrá solicitar la convocatoria inmediata del Consejo Permanente para realizar una apreciación colectiva de la situación y adoptar las decisiones que estime convenientes. Se agrega en el referido artículo que, si las gestiones diplomáticas promovidas por la OEA fracasaran, o la urgencia del caso lo aconsejare, el Consejo Permanente convocará de inmediato a un período extraordinario de sesiones de la Asamblea General para que esta adopte las decisiones que estime apropiadas. Sin embargo, no se señala específicamente cuáles serían estas decisiones, salvo, nuevamente, las gestiones diplomáticas.

El hecho concreto es que «la alteración del orden constitucional que afecte gravemente el orden democrático en un Estado miembro» es, ante todo, una definición política. Como señaló Maxwell A. Cameron en una conferencia internacional sobre la CDI, citando al jurista peruano Diego García Sayán, la decisión sobre qué tipo de situaciones afectan gravemente el orden democrático es un juicio de naturaleza política, no un juicio académico; por ello, los académicos solo podrían aportar evidencia empírica sobre el estado de la democracia basados en indicadores. Cameron añade que «para que se pueda aplicar la Carta, tiene que haber fundamentos sólidos que nos permitan concluir que este o aquel país no es una democracia, que no se respeta la separación de poderes y que el sistema electoral es fraudulento o, simplemente, está tan degradado que no puede ser avalado por parte de la comunidad internacional, como fue el caso del Perú en el año 2000» (2011, p. 33). En tal sentido, este autor considera que es importante tener una definición precisa de lo que significa «alteración del orden democrático».

Por otra parte, Cameron refiere que la CDI es débil porque sus mecanismos de cumplimiento son débiles; y son así porque la OEA es básicamente un club de Estados o, más precisamente, un club de ejecutivos, en el sentido de que sus miembros solo son los poderes ejecutivos de los Estados (p. 32). Esta es la razón de que las decisiones sean esencialmente políticas. En general, observamos que las acciones preventivas y correctivas solo podrían ser impulsadas por los Estados

LAS DEMOCRACIAS

CON LIBERTADES

DISMINUIDAS EN

LATINOAMÉRICA

EN EL SIGLO

XXI Y LA CARTA

DEMOCRÁTICA

INTERAMERICANA:

¿DOS MODELOS

DE DEMOCRACIA

EN LA REGIÓN?

$21^{\text {ST }}$ CENTURY

ILLIBERAL

DEMOCRACIES IN

LATIN AMERICA

AND THE INTER-

AMERICAN

DEMOCRATIC

CHARTER:TWO

MODELS OF

DEMOCRACY IN

THE REGION? 
miembros, esto es, por los jefes de Estado o jefes de gobierno de los países de las Américas.

En suma, en la CDI tenemos una amplia definición de democracia -nuestro edificio multinivel - pero un cuello de botella en sus órganos de cumplimiento, controlados por presidentes, embajadores o ministros de relaciones exteriores, todos ellos como parte del Poder Ejecutivo de sus respectivos países. Tal vez por esta razón, desde la academia y la sociedad civil, se expresa la necesidad de implementar reformas institucionales dentro de la OEA y de reformar la CDI para permitir, por ejemplo, que los otros poderes del Estado - Congreso y Poder Judicial-, las entidades de accountability horizontal de encargo o mandato - como las Defensorías del Pueblo-, organismos no gubernamentales (ONG) o partidos de oposición puedan ser escuchados por el secretario general, el Consejo Permanente o incluso la Asamblea General. Sin embargo, la Realpolitik está presente en la OEA. En efecto, por ejemplo, como se ha podido constatar recientemente, el Consejo Permanente, por mayoría de votos, denegó el pedido de una lideresa de la oposición política venezolana, María Corina Machado, para hablar en una sesión de este órgano sobre la situación política de su país. iEs la OEA, a pesar de sus defectos, la única alternativa para fortalecer y preservar la democracia en América Latina?

\section{IV.LA UNASUR Y LA DEFENSA DE LAS DEMOCRACIAS SUDAMERICANAS}

La Unión de Naciones Suramericanas (UNASUR) es una organización intergubernamental creada como consecuencia del tratado firmado en el año 2008 por Argentina, Bolivia, Brasil, Colombia, Chile, Ecuador, Guyana, Paraguay, Perú, Surinam, Uruguay y Venezuela.

De acuerdo con el artículo 2 del referido Tratado Constitutivo, el objetivo de la UNASUR es

[...] construir, de manera participativa y consensuada, un espacio de integración y unión en lo cultural, social, económico y político entre sus pueblos, otorgando prioridad al diálogo político, las políticas sociales, la educación, la energía, la infraestructura, el financiamiento y el medio ambiente, entre otros, con miras a eliminar la desigualdad socioeconómica, lograr la inclusión social y la participación ciudadana, fortalecer la democracia y reducir las asimetrías en el marco del fortalecimiento de la soberanía e independencia de los Estados.

En el año 2010, los Estados miembros de la UNASUR suscribieron el Protocolo Adicional al Tratado Constitutivo sobre Compromiso con la Democracia, que entró en vigencia el 19 de marzo de 2014. 
Su artículo 1 señala que dicho protocolo «se aplicará en caso de ruptura o amenaza de ruptura del orden democrático, de una violación del orden constitucional o de cualquier situación que ponga en riesgo el legítimo ejercicio del poder y la vigencia de los valores y principios democráticos». Asimismo, el artículo 4 establece las siguientes medidas si se producen los eventos referidos en el artículo 1 :

a. Suspensión del derecho a participar en los distintos órganos e instancias de la UNASUR, así como del goce de los derechos y beneficios conforme al Tratado Constitutivo de la UNASUR.

b. Cierre parcial o total de las fronteras terrestres, incluyendo la suspensión y/o limitación del comercio, tráfico aéreo y marítimo, comunicaciones, provisión de energía, servicios y suministros.

c. Promover la suspensión del Estado afectado en el ámbito de otras organizaciones regionales e internacionales.

d. Promover, ante terceros países y/o bloques regionales, la suspensión de los derechos y/o beneficios del Estado afectado, derivados de los acuerdos de cooperación de los que fuera parte.

e. Adopción de sanciones políticas y diplomáticas adicionales (UNASUR, 2010).

Jorge Santistevan, el primer defensor del Pueblo del Perú, comentando una ponencia presentada por Jennifer McCoy en la misma conferencia sobre la CDI en la que intervino Maxwell A. Cameron (cuya intervención se cita líneas arriba), refirió que, a primera vista, el protocolo pareciera ser más completo que la CDI. Sin embargo, advirtió que si la UNASUR se convirtiera en un club de autodefensa de los titulares del Poder Ejecutivo, «no sería viable la cláusula democrática de UNASUR para hacer frente a las amenazas que se producen desde el Ejecutivo contra otros poderes del Estado o contra los órganos constitucionales autónomos» (Santistevan, 2011, p. 20).

El desempeño de la UNASUR en algunos eventos políticos de la región pareciera confirmar el argumento de Santistevan. Por ejemplo, en la sesión extraordinaria realizada el 19 de abril de 2013 en Lima, el Consejo de Jefes de Estado y de Gobierno de la UNASUR respaldó los resultados de la elección presidencial venezolana que se llevó a cabo el 14 de abril de 2013 y que puso al vicepresidente Nicolás Maduro en la presidencia. Como sabemos, esa elección se llevó a cabo en medio de una delicada situación política generada como consecuencia de la muerte del presidente venezolano Hugo Chávez el 5 de marzo de 2013.

Asimismo, en respuesta a la violenta situación política ocurrida en Venezuela, los ministros de relaciones exteriores de la UNASUR aprobaron una resolución el 12 de marzo de 2014, mediante la cual acordaron 
«respaldar los esfuerzos del Gobierno de la República Bolivariana de Venezuela para propiciar un diálogo entre el Gobierno, todas las fuerzas políticas y actores sociales con el fin de lograr un acuerdo que contribuya al entendimiento y la paz social». La mencionada resolución también designó, a solicitud del gobierno venezolano, una comisión de ministros de relaciones exteriores de la UNASUR que acompañe, apoye y asesore el diálogo político en ese país (UNASUR, 2014).

Con el transcurso del tiempo podremos saber si este club podrá convertirse en una institución internacional que promueva y defienda efectivamente la democracia en la región.

\section{CONCLUSIONES}

En hojas de papel, es decir en constituciones y acuerdos internacionales, los países de Latinoamérica tienen regímenes democráticos en buena forma. Sin embargo, comoseñaló Robert A. Dahl, «ninguna Constitución [a lo que agregamos también ningún instrumento internacional] puede asegurar la democracia en un país donde las condiciones favorables a ella están ausentes» (Dahl, 2003, p. 142). En este tipo de países, podemos encontrar más constituciones nominales y semánticas que normativas, conforme a la clásica tipología de Karl Loewenstein, donde las primeras y las segundas siempre alardean de ser normativas. Es por ello que Loewenstein afirma que es necesario penetrar en la realidad del proceso político (1979, p. 219).

Después de analizar varios procesos políticos en América Latina, más allá de las constituciones y los tratados, encontramos dos tendencias: democracias que descansan en la tradición liberal, y democracias con libertades disminuidas que se preocupan esencialmente de los derechos económicos, sociales y culturales. El problema radica en que las democracias liberales a menudo se contentan con un edificio de un solo piso, sin espacio para satisfacer las necesidades de los ciudadanos; mientras que, por su parte, las democracias con libertades disminuidas desean erigir un sólido segundo piso sobre un primer piso construido en la arena.

Finalmente, encontramos que entidades intergubernamentales como la OEA y la UNASUR no cuentan con instrumentos efectivos y/o voluntad política para hacer cumplir los acuerdos que promuevan una democracia «totalmente equipada», necesaria para dejar, de una vez por todas, el siglo XX (o incluso el siglo XIX). 
VI. BIBLIOGRAFÍA

Cameron, Maxwell (2014). The Myth of Competitive Authoritarianism in the Andes. Manuscrito. 13 de febrero de 2014. Expreso mi gratitud al profesor Sinesio López de la PUCP por haberme proporcionado una copia de este artículo y haberme autorizado, en nombre del autor, a utilizarlo en este trabajo.

Cooper, Andrew (2004). The making of the Inter-American Democratic Charter: A case of complex multilateralism. International Studies Perspectives, 5(1), 92-113.

Dahl, Robert (1989). Democracy and Its Critics. New Haven/Londres: Yale University Press.

Dahl, Robert (2003). How Democratic is the American Constitution? New Haven/ Londres: Yale University Press.

Legler, Thomas (2011). Lecciones de la crisis hondureña. En Santiago Mariani (ed.), La democracia en el marco de la Carta Democrática Interamericana (pp. 4245). Lima: IDEA International.

Levitsky, Steven \& Lucan Way (2010). Competitive Authoritarianism. Cambridge: Cambridge University Press.

Loewenstein, Karl (1979). Teoría de la Constitución. Barcelona/Caracas/México: Ariel.

Mariani, Santiago (ed.) (2011). La democracia en el marco de la Carta Democrática Interamericana. Lima: IDEA International.

Møller, Jørgen (2008). A Critical Note on «The Rise of Illiberal Democracy». Australian Journal of Political Science, 43 (3), 555-561.

O’Donnell, Guillermo (1994). Delegative Democracy. Journal of Democracy, 5 (1), 55-69.

O'Donnell, Guillermo (2003). Horizontal Accountability: The Legal Institutionalization of Mistrust. En Scott Mainwaring \& Christopher Welna (eds.), Democratic Accountability in Latin America (pp. 34-54). Oxford: Oxford University Press.

Organización de los Estados Americanos (OEA) (2001). Carta Democrática Interamericana. Lima, 11 de setiembre de 2001. Recuperado de http://www. oas.org/OASpage/esp/Documentos/Carta_Democratica.htm.

Perina, Rubén (2012). The Inter-American Democratic Charter: An Assessment and Ways to Strengthen It. En The Road to Hemispheric Cooperation: Beyond the Cartagena Summit of the Americas (pp. 77-87). Washington D.C.: The Brooking Institution.

Peruzzotti, Enrique \& Catalina Smulovitz (2002). Accountability social: la otra cara del control. En Entique Peruzzotti \& Catalina Smulovitz (eds.) Controlando la política (pp. 23-52). Buenos Aires: Temas.

Santistevan, Jorge (2011). Comentario de Jorge Santistevan. Comentario a la exposición de Jennifer McCoy en el Primer panel: Las respuestas internacionales

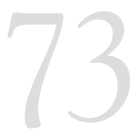

LAS DEMOCRACIAS

CON LIBERTADES

DISMINUIDAS EN

LATINOAMÉRICA

EN EL SIGLO

XXI Y LA CARTA

DEMOCRÁTICA

INTERAMERICANA:

¿DOS MODELOS

DE DEMOCRACIA

EN LA REGIÓN?

$21^{\text {ST }}$ CENTURY

ILLIBERAL

DEMOCRACIES IN

LATIN AMERICA

AND THE INTER-

AMERICAN

DEMOCRATIC

CHARTER:TWO

MODELS OF

DEMOCRACY IN

THE REGION? 
frente a las crisis democráticas en las Américas. En Santiago Mariani (ed.), La democracia en el marco de la Carta Democrática Interamericana (pp. 17-21). Lima: IDEA International.

Schrijver, Nico (2011). Paving the Way Towards... One Worldwide Human Rights Treaty!. Netherlands Quarterly of Human Rights, 29 (3), 257-261.

Touraine, Alaine (2006). ¿Qué es la democracia? México, D.F: Fondo de Cultura Económica.

Unión de Naciones Suramericanas (UNASUR) (2008). Tratado Constitutivo de la Unión de Naciones Suramericanas. Recuperado de http://www.unasursg. org/images/descargas/DOCUMENTOS\%20CONSTITUTIVOS\%20DE\%20 UNASUR/Tratado-UNASUR-solo.pdf.

Unión de Naciones Suramericanas (UNASUR) (2010). Protocolo adicional al Tratado Constitutivo de UNASUR sobre Compromiso con la Democracia. Recuperado de http://www.unasursg.org/images/descargas/ DOCUMENTOS\%20CONSTITUTIVOS\%20DE\%20UNASUR/ProtocoloAdicional-al-Tratado-Constitutivo-de-UNASUR-sobre-Compromiso-con-laDemocracia-opt.pdf

Unión de Naciones Suramericanas (UNASUR) (2014). UNASUR/CMRE/ RESOLUCIÓN N 2/2014. Santiago de Chile, 12 de marzo de 2014. Recuperado de https://repo.unasursg.org/alfresco/service/unasursg/documents/ content? noderef $=26 \mathrm{c} 42049-01 \mathrm{db}-4300-88 \mathrm{~b} 6-337485 \mathrm{~b} 9 \mathrm{~d} 63 \mathrm{~d}$.

Zakaria, Fareed (1997). The Rise of Illiberal Democracy. Foreign Affairs, 76 (6), $22-43$. 